\title{
EVOLUCIÓN HISTÓRICA DEL MARCO INTERNACIONAL DE LA PROTECCIÓN JURÍDICA DEL MEDIO AMBIENTE
}

HISTORICAL EVOLUTION OF THE INTERNATIONAL FRAMEWORK OF LEGAL PROTECTION OF THE ENVIRONMENT

MiLLITZA Franciskovic IngUNZA Nuccia Seminario Hurtado

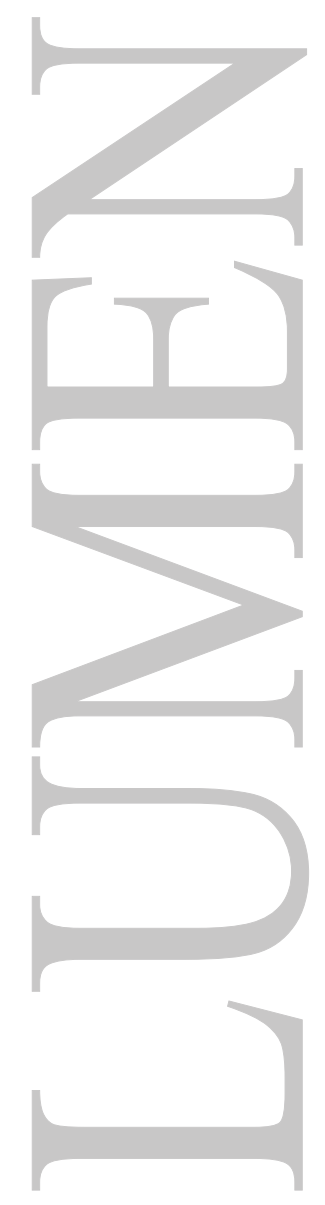




\title{
EVOLUCIÓN HISTÓRICA DEL MARCO INTERNACIONAL DE LA PROTECCIÓN JURÍDICA DEL MEDIO AMBIENTE
}

\author{
HISTORICAL EVOLUTION OF THE INTERNATIONAL FRAMEWORK \\ OF LEGAL PROTECTION OF THE ENVIRONMENT
}

Millitza Franciskovic Ingunza

Nuccia Seminario Hurtado

\begin{abstract}
RESUMEN:
El presente artículo tiene como objetivo desarrollar el marco histórico del tratamiento jurídico internacional del Derecho Ambiental; asimismo, analizar los objetivos y resultados que enmarcaron en el marco jurídico internacional ambiental, además desarrolla los problemas medioambientales del planeta que están íntimamente relacionados con las condiciones económicas y los problemas de justicia social, demostrándose que las necesidades sociales, medioambientales y económicas se deben equilibrar las unas con las otras para obtener resultados sostenibles a largo plazo, destaca que los problemas medioambientales del planeta estaban íntimamente relacionados con las condiciones económicas y los problemas de justicia social, demostrándose que las necesidades sociales, medioambientales y económicas se deben equilibrar las unas con las otras para obtener resultados sostenibles a largo plazo
\end{abstract}

\section{PALABRAS CLAVES:}

Derecho ambiental, derecho internacional público, derecho internacional ambiental, protección jurídica, convenciones, tratados.

\section{ABSTRACT:}

The objective of this article is to develop the historical framework of the international legal treatment of Environmental Law, as well as to analyze the objectives and results that framed the international environmental legal framework, as well as to develop the environmental problems of the planet that are intimately related to economic conditions and The problems of social justice, demonstrating that social, environmental and economic needs must be balanced with each other to obtain sustainable results in the long term, stresses that the environmental problems of the planet were intimately related to economic conditions and problems of justice social, demonstrating that social, environmental and economic needs must be balanced with each other to obtain long-term sustainable results

\section{KEY WORDS:}

Environmental law, public international law, international environmental law, legal protection, conventions, treaties.

\section{INTRODUCCIÓN.-}

El medio ambiente es un pilar imprescindible para el desarrollo progresivo de los seres vivientes en el planeta tierra, constituyéndose de carácter fundamental la protección de éste. Por ello la Comunidad Internacional se ha visto en la necesidad de establecer instrumentos jurídicos internacionales que planteen como objetivo principal garantizar, perseverar y amparar el medio ambiente en el planeta tierra.

De esa manera, a lo largo de la historia, la protección internacional del medio ambiente se plasma a través de una evolución -histórica- y - jurídica- a raíz de los dilemas negativos medio ambientales que arrasaban al planeta tierra. Por ello, se constituye de carácter imprescindible el desarrollo normativo que tiene como característica principal la internacionalización del medio ambiente. 
Finalmente, en el planteamiento del presente artículo nos enfocaremos en el desarrollo progresivo de los instrumentos ambientales-internacionales enmarcados en la historia del derecho internacional ambiental, a su vez, el concepto y los resultados que destacaron en la Comunidad Internacional.

\section{1) CONCEPTOS PRELIMINARES}

\subsection{Derecho Ambiental}

Es el conjunto de normas y principios de acatamiento imperativo, elaborados con la finalidad de regular las conductas humanas para lograr el equilibrio entre las relaciones del hombre y el ambiente al que pertenece, a fin de lograr un ambiente sano y el desarrollo sostenible.(Andaluz, 2006, p.505)

Según Jaquenod (1999), "el derecho ambiental asume una problemática multi e interdisciplinaria, pues los diversos sectores que la integran, interaccionan e interdependen entre sí. Adquiere trascendencia como disciplina científica, cuando se comprende que el entorno constituye un todo y sus diferentes componentes se encuentran en permanente interacción". (p.35)

El derecho ambiental reúne una serie de peculiaridades, como:

a) Es interdisciplinario, porque en él se yuxtapone una amplia gama de disciplinas más o menos relacionadas. Esta interdisciplinariedad se articula dentro de un marco de importación de conceptos, procedimientos, metodologías, aplicándose técnicas de un campo y adaptándolas a otros. (Jaquenod, 1999, p58.)

b) Es preventivo, en materia ambiental son necesarias aquellas acciones que se anticipan a prevenir, cualquier tipo de degradación ambiental, en lugar de limitarse a verificar, e intentar a posteriori reparar los daños ambientales.

La coacción a posteriori resulta ineficaz en Derecho Ambiental por cuanto de haberse producido ya las consecuencias biológicas y socialmente nocivas, la represión podrá tener una trascendencia moral, pero difícilmente compensará los graves daños ocasionados al ambiente.

c) Es sistémico, en cuanto que la regulación de conductas no se realiza aisladamente, sino teniendo en cuenta el comportamiento de los elementos naturales y las interacciones en ellos determinadas como consecuencia de las actuaciones del hombre.

d) Es de carácter colectivo, aunque al logro de sus objetivos puedan concurrir normas de otra naturaleza, como son aquellas que regulan las distintas relaciones o situaciones de vecindad, las cuales sólo muy localizadamente pueden coadyuvar a estos objetivos. Los conflictos que aborda enfrentan a amplios colectivos: productores y consumidores, contaminadores y contaminados, industriales entre sí, propugnadores del consumo y defensores de la calidad de vida, entre otros.

e) Carácter supranacional o transfronterizo, porque los problemas ambientales pueden rebasar las fronteras regionales, estatales y continentales y se destaca la importancia de la cooperación internacional, como el comportamiento de los recursos no tiene en cuenta fronteras, los efectos negativos producidos en un país determinado pueden afectar a los recursos de otros países, sea esto de modo directo o indirecto, y/o en el corto, mediano, largo plazo.

Rigurosa regulación técnica, la normativa del Derecho Ambiental contiene prescripciones rigurosamente técnicas, que determinan las condiciones precisas en que deben realizarse las 
actividades afectadas.(Bustamante, p.50) La discrecionalidad de la administración pública y la propia labor del jurista se encuentran limitadas en el marco técnicamente precisado para la regulación de conductas, aunque la prescripciones técnicas deben ser entendidas como aquellas que marcan límites y umbrales, que permiten modulaciones y apreciaciones cuando se entrecruzan factores diversos que se resisten a un planteamiento puramente científico y matemático.

\subsection{Derecho Internacional Ambiental}

La protección del medio ambiente es una disciplina que se desprende del derecho internacional público. Las fuentes regulatorias relativas están constituidas por declaraciones, convenciones, tratados y entre otros instrumentos jurídicos de carácter internacional, que están abocados a la prevención, reducción o reparación de daños ambientales causados al Estado en el territorio de otros.

Foy y Novak (2003) expresan que "se suele afirmar que el Derecho Ambiental es, por esencia, un Derecho Internacional. Ello debido a que sus orígenes, como corpus disciplinario, y sus fuentes histórico-materiales se identifican con procesos signados por su relevancia y enfoque internacional con relación a determinados problemas de orden material. (p.33)

El derecho internacional ambiental es una rama del derecho internacional público, cuerpo normativo creado por los Estados para que estos gobiernen los problemas que surgen entre ellos; está enfocado en los temas como la población, la biodiversidad, el cambio climático, el agotamiento de la capa de ozono, las sustancias tóxicas y peligrosas, la contaminación del aire, tierra, mar y agua transfronteriza, la conservación de los recursos marinos, la desertificación y el daño nuclear.

Sobre las fuentes del derecho internacional ambiental, el notable ambientalista Russell (2014) afirma que "son las mismas que las de derecho internacional general: convenciones internacionales, costumbres internacionales, principios generales del derecho y escritos de juristas altamente cualificados." (p.3)

\section{2) EVOLUCIÓN HISTÓRICA DEL MARCO INTERNACIONAL DE LA PROTECCIÓN JURÍDICA DEL MEDIO AMBIENTE}

\subsection{Conferencia de Estocolmo (1972)}

\subsubsection{Definición}

La Conferencia de Estocolmo es un conjunto de 106 Recomendaciones y 26 Principios que abogan por el derecho al desarrollo sostenible y a la protección y conservación del medio ambiente. Llevada a cabo en Estocolmo, Suecia del 05 al 16 de junio de 1972 asistieron 113 países, participando el Perú y se debate por primera vez en un foro internacional la problemática del medio ambiente, haciendo resaltar la importancia del mismo para el ser humano y los demás seres vivos.

La Universidad de Melbourne (2018) señala que "esta declaración representó un primer intento importante de considerar el impacto humano global en el medio ambiente y un intento internacional para enfrentar el desafío de preservar y mejorar el medio ambiente humano. La Declaración de Estocolmo adoptó objetivos y metas de política ambiental en su mayoría en lugar de posiciones normativas detalladas".

El ilustre ambientalista Fajardo (2015) expresa que:

"A pesar de que la Declaración de Estocolmo abordó el problema de la contaminación doméstica y transnacional, no lo calificó como un posible delito ambiental, sino simplemente 
como una preocupación que los Estados debían enfrentar a través de una acción cooperativa y un mayor desarrollo del "derecho internacional sobre responsabilidad e indemnización". Para las víctimas de la contaminación y otros daños ambientales causados por actividades dentro de la jurisdicción o el control de dichos Estados." (p.9)

\subsubsection{Resultado de Estocolmo}

Esta Conferencia es importante porque exhorta por primera vez a los Estados que las Recomendaciones y Principios deben ser introducidos en el ordenamiento jurídico de cada Estado; problemas medioambientales del planeta estaban íntimamente relacionados con las condiciones económicas y los problemas de justicia social, demostrándose que las necesidades sociales, medioambientales y económicas se deben equilibrar las unas con las otras para obtener resultados sostenibles a largo plazo. Pone énfasis - desde estos tiempos iniciáticos - sobre la manera de generar instituciones aptas para el logro de políticas concertadas. Integración, coordinación, prevención y restauración son elementos que surgen desde esta Convención, primer hito de la disciplina en el camino a la sustentabilidad.

Según Atilio (2007) sobre el resultado plantea que:

"Puede ser caracterizada durante un primer periodo por el bosquejo y la adopción de instrumentos internacionales que regulan amplios sectores del medio ambiente: océanos, aguas interiores, aire, suelo y vida silvestre. En una segunda etapa emerge un nuevo campo de regulación transversal, que incluye productos y desechos tóxicos o peligrosos, radioactividad, desechos nucleares y actividades peligrosas. En una tercera etapa los temas preponderantes son la prevención de la disminución de la capa de ozono, así como también el calentamiento global y la salvaguardia de la diversidad biológica." (p.276.)

La Conferencia de Estocolmo enuncia en su preámbulo que: los dos aspectos del medio humano, el natural y el artificial, son esenciales para el bienestar del hombre y para el goce de los derechos fundamentales, incluso el derecho a la vida misma (párrafo 1); la protección y mejoramiento del medio humano es una cuestión fundamental que afecta al bienestar de los pueblos y al desarrollo económico del mundo entero, un deseo urgente de todos los pueblos del mundo y un deber de todos los gobiernos (párrafo 2). A partir de esta Conferencia, la preservación del medio ambiente queda incorporada como un objetivo más a conseguir por el Derecho Internacional.

\subsection{Conferencia de Río (1992)}

\subsubsection{Definición}

Es un instrumento de carácter jurídicamente no vinculante, sus previsiones, que suelen ser más concretas que las de la Declaración de Estocolmo, proporcionan un marco general para el desarrollo del Derecho ambiental a nivel nacional e internacional que constituye un importante punto de referencia para la toma de decisiones en este ámbito. (Atilio, 2007, p.35) Su principal objetivo fue desarrollar un verdadero Derecho Ambiental trasnacional, ubicando cada país dentro de su realidad social, económica, política y ambiental, con énfasis especial en las obligaciones y derechos específicos de los países en desarrollo. (Lozano, p.58-59).

Instituto de las Naciones Unidas para la Formación e Investigación (2006) expresa que:

Declaración de Río, es un documento breve que consta de 27 principios, tiene un carácter compuesto. Reafirma los principios de la Declaración de Estocolmo de 1972 en los que se basa, pero su enfoque y filosofía son muy diferentes. El concepto central es el desarrollo 
sostenible, que significa la integración del desarrollo y la protección del medio ambiente según lo define la Comisión Mundial sobre el Medio Ambiente y el Desarrollo. El Principio 4 es importante a este respecto: establece que para alcanzar los objetivos del desarrollo sostenible, la protección ambiental debe ser una parte integral del proceso de desarrollo y no puede considerarse de manera aislada. (p.34)

Seguidamente, el notable ambientalista Strong (2001) señala que:

"Se le conoce también como ECO-92 o Cumbre de la Tierra y procura acercar las posturas de los países del Norte y del Sur, que se ubicaban en los extremos de la protección del ser humano, se reconoce que los países industrializados son en gran parte responsables de los riesgos ambientales y han sido los beneficiados de la riqueza que se ha acumulado a través de los procesos de crecimiento económico." (Pp. 1- 9.)

La Cumbre de la Tierra marcó un hito porque centró la atención mundial en la idea de que los problemas medioambientales del planeta estaban íntimamente relacionados con las condiciones económicas y los problemas de justicia social, demostrándose que las necesidades sociales, medioambientales y económicas se deben equilibrar las unas con las otras para obtener resultados sostenibles a largo plazo.

\subsubsection{Resultados}

Hay un reconocimiento de las características globales y multidimensionales de los problemas ambientales y de los remedios potenciales; la mayoría de los Estados ya aceptan que se requieren esfuerzos globales para resolver varios aspectos del deterioro ambiental, como contaminación de océanos, reducción de la capa de ozono, el efecto invernadero y las amenazas a la biodiversidad. Otro avance importante es lo que concierne a los ecosistemas que han sido ampliados debido a los acercamientos de integración para salvaguardar el medio ambiente del planeta. Se incorpora la protección de la flora y la fauna silvestre, se incluye esfuerzos para revertir tendencias hacia la agricultura monocultural y ganadería.

Seguido a ello, Atilio (2007) expresa que "también hay un reconocimiento en aumento del rol crucial de fuerzas y actores económicos en la protección del medio ambiente. Las empresas se han vuelto más abiertas a la presión pública, a los requerimientos de seguridad y a una desarrollada conciencia ambiental." (pp. 284-285)

Se obtuvo los siguientes documentos:

\section{a) Convención sobre biodiversidad}

El Convenio sobre la Diversidad Biológica es el primer acuerdo mundial que protege todos los aspectos de la biodiversidad. EI CDB, que es un convenio legalmente vinculante, fue adoptado en la Cumbre de la Tierra en 1992 en Río de Janeiro, Brasil, y entró en vigor a finales de 1993. Se propone como objetivos: 1) proteger la biodiversidad, 2) usar los recursos biológicos de manera sostenible, 3) compartir equitativamente los beneficios de estos recursos.

Bustamante (1995) expresa que:

El Convenio de Biodiversidad requiere de los países miembros: elaborar estrategias para la conservación y la utilización sostenible de la biodiversidad, identificar hábitats y especies críticas y establecer programas de seguimiento, establecer áreas protegidas para estos hábitats y especies, regular y dar seguimiento a las actividades que puedan amenazar la 
biodiversidad, cooperar con otros estados en la educación, el entrenamiento y la investigación para proteger la biodiversidad. (p. 34)

El cumplimiento del Convenio está condicionado por el tipo de disposiciones que contiene, compuesto por normas flexibles, que evita el uso de palabras que manifiesten una obligación de resultado. Así, recurre a los verbos promover, fomentar, procurar, facilitar, etc., los cuales sugieren más un comportamiento que el cumplimiento imperativo de las normas contenidas en él. Más aún, si encontramos locuciones como: en la medida de lo posible y según proceda o con arreglo a sus condiciones y capacidades particulares, que aunque podría interpretarse como el respeto a las capacidades económicas y ambientales de cada Estado miembro, puede también debilitar su contenido obligatorio.

\section{b) Convención sobre Cambio Climático}

Fue firmada en New York por 155 Estados y la Comunidad Europea el 09 de mayo de 1992, entrando en vigor en 1994. Su objetivo es combatir el cambio climático mediante la adopción de medidas tendientes a estabilizar las emisiones de gases de efecto invernadero en la atmósfera. La adopción de medidas por la Comunidad Internacional para la consecución del objetivo, se basa en el principio de precaución, que impele a los Estados a adoptar medidas para prevenir el calentamiento de la tierra y mitigar sus efectos. Al suscribir la Convención, los países desarrollados acordaron reducir sus emisiones de dióxido de carbono y otros "gases de efecto invernadero" a los niveles de 1990 antes del fin del año 2000. Esos países, que son responsables del $60 \%$ de las emisiones anuales de dióxido de carbono, convinieron también en transferir a los países en desarrollo tecnología e información que los ayudaran a hacer frente a los problemas derivados del cambio climático.

En 1995 se decidió negociar un Protocolo que contuviese medidas de reducción vinculantes de las emisiones de GEI (gases efecto invernadero) para el periodo posterior al año 2000, celebrándose en Kioto (Japón), por lo que se conoce como "Protocolo de Kioto"; viene a reforzar el cumplimiento de los objetivos de la Convención, puesto que a través de él las partes se comprometen a lograr objetivos individuales y jurídicamente vinculantes. (Lozano, pp.548-551.)

c) Declaración de principios sobre bosques o "Convención Internacional de lucha contra la desertificación en los países afectados por sequía o grave desertificación, en particular en África"

La Convención tiene como objetivo principal el promover una acción efectiva a través programas locales y cooperación internacional, estableciendo las pautas para luchar contra la desertificación y disminuir los efectos de la sequía en los países afectados, a través de: el mejoramiento de la productividad del suelo, la rehabilitación del suelo, la conservación y ordenación de los recursos de las tierras y los recursos hídricos. La Convención consta de 49 artículos y de 5 anexos con disposiciones de aplicación para África, Asia, América Latina y el Caribe, el Mediterráneo septentrional y Europa Central y Oriental. La aplicación de la Convención requiere una vigorosa cooperación internacional entre países desarrollados y países en desarrollo. No obstante, los Gobiernos de los países afectados por la desertificación son los responsables de crear un entorno que permita que las propias poblaciones locales pongan fin al proceso de degradación de las tierras. La Convención fue firmada en 1994 y entró en vigor el 26 de diciembre de 1996. Hasta ahora, más de 170 países han ratificado la Convención, que es un instrumento jurídicamente vinculante. ${ }^{1}$

\section{d) Declaración de Río sobre Medio Ambiente y Desarrollo}

Los principios consagrados en la Declaración de Río no tienen la calidad de normas vinculantes, pero sirven de inspiración al legislador al crear normas, o para la interpretación normativa aclarando el sentido de la ley o imputándole contenido valorativo ante una laguna jurídica. (Andaluz, 2006, p.579)

\footnotetext{
${ }^{1}$ La Unión Europea. Recuperado en: https://europa.eu/european-union/index es
} 
La Declaración consiste en un listado de 27 principios que relacionan y concilian el desarrollo económico y social con la protección del sistema ambiental mundial.

Se puede sintetizar el contenido de cada principio de la siguiente manera:

\begin{tabular}{|c|c|}
\hline Principio 1 & $\begin{array}{l}\text { Derecho de las personas a una vida } \\
\text { saludable (consagra un derecho } \\
\text { fundamental al desarrollo y recuerda que: } \\
\text { los seres humanos constituyen el centro } \\
\text { de las preocupaciones relacionadas con } \\
\text { el desarrollo sostenible). }\end{array}$ \\
\hline Principio 2 & $\begin{array}{l}\text { Responsabilidad de los Estados de no } \\
\text { causar daño en el medio ambiente de } \\
\text { otros Estados. }\end{array}$ \\
\hline Principio 3 & $\begin{array}{l}\text { La preocupación por las generaciones } \\
\text { presentes y futuras. }\end{array}$ \\
\hline Principio 4 & $\begin{array}{l}\text { Protección del medio ambiente unido al } \\
\text { desarrollo sostenible. }\end{array}$ \\
\hline Principio 5 & $\begin{array}{l}\text { Erradicar la pobreza para lograr el } \\
\text { desarrollo sostenible. }\end{array}$ \\
\hline Principio 6 & $\begin{array}{l}\text { La prioridad de los países más } \\
\text { vulnerables ambientalmente. }\end{array}$ \\
\hline Principio 7 & $\begin{array}{l}\text { Estados tienen responsabilidades } \\
\text { comunes pero diferenciadas. }\end{array}$ \\
\hline Principio 8 & $\begin{array}{l}\text { Eliminar el sistema de producción y } \\
\text { consumo insostenibles. }\end{array}$ \\
\hline Principio 9 & $\begin{array}{l}\text { Estados deben } \quad \text { intercambiar } \\
\text { conocimientos científicos y tecnológicos. }\end{array}$ \\
\hline Principio 10 & Participación de todos los ciudadanos. \\
\hline Principio 11 & $\begin{array}{l}\text { Se deben dictar leyes ambientales } \\
\text { eficaces. }\end{array}$ \\
\hline Principio 12 & $\begin{array}{l}\text { Consenso internacional en la solución } \\
\text { de problemas. }\end{array}$ \\
\hline Principio 13 & $\begin{array}{l}\text { Responsabilidad e indemnización por } \\
\text { los daños ambientales. }\end{array}$ \\
\hline Principio 14 & $\begin{array}{l}\text { Cooperación entre Estados para evitar } \\
\text { actividades que causen daño ambiental. }\end{array}$ \\
\hline Principio 15 & $\begin{array}{l}\text { Aplicación del criterio de precaución } \\
\text { para proteger el ambiente. }\end{array}$ \\
\hline Principio 16 & $\begin{array}{l}\text { El que contamina asume costos de daño } \\
\text { ambiental. }\end{array}$ \\
\hline Principio 17 & Evaluación del impacto ambiental. \\
\hline Principio 18 & $\begin{array}{l}\text { Información y asistencia entre Estados } \\
\text { en emergencias ambientales. }\end{array}$ \\
\hline
\end{tabular}




\begin{tabular}{|l|l|}
\hline Principio 19 & $\begin{array}{l}\text { Brindar información oportuna sobre } \\
\text { efectos nocivos ambientales. }\end{array}$ \\
\hline Principio 20 & $\begin{array}{l}\text { El rol fundamental de las mujeres en el } \\
\text { desarrollo sostenible. }\end{array}$ \\
\hline Principio 21 & $\begin{array}{l}\text { La labor de los jóvenes en el desarrollo } \\
\text { sostenible. }\end{array}$ \\
\hline Principio 22 & $\begin{array}{l}\text { El rol de los pueblos indígenas en la } \\
\text { ordenación del medio ambiente. }\end{array}$ \\
\hline Principio 23 & $\begin{array}{l}\text { Protección del medio ambiente de } \\
\text { pueblos sometidos. }\end{array}$ \\
\hline Principio 24 & $\begin{array}{l}\text { Protección del medio ambiente en época } \\
\text { de conflicto armado. }\end{array}$ \\
\hline Principio 25 & $\begin{array}{l}\text { La fusión de la paz, el desarrollo y medio } \\
\text { ambiente. }\end{array}$ \\
\hline Principio 26 & $\begin{array}{l}\text { Solución de conflictos por medios } \\
\text { pacíficos. }\end{array}$ \\
\hline Principio 27 & $\begin{array}{l}\text { Cooperación de buena fe para } \\
\text { aplicación de principios. }\end{array}$ \\
\hline
\end{tabular}

Elaboración propia

Dicha Declaración reconoce la necesidad de formulación de instrumentos legales tanto a nivel nacional como internacional que regulen de manera adecuada la protección del medio ambiente (Principios 11 y 13). Reconociendo los impactos que sobre el medio ambiente tiene el desarrollo económico, se señala la necesidad de un sistema económico internacional que permita el crecimiento económico y desarrollo sostenible de todos los países y donde las disposiciones comerciales con fines ambientales no constituyan un medio de discriminación ni una barrera para el comercio internacional (Principio 12). En este mismo sentido, se exhorta a los países a internalizar los costos ambientales y crear instrumentos económicos partiendo del principio de que el que contamina paga. (Principio 16).

Asimismo, la Declaración establece como medidas de cautela para la protección ambiental, la aplicación del principio de precaución (Principio 15) y la evaluación de impactos ambientales (Principio 17), cuando haya riesgo de daños considerables al medio ambiente. Se reconoce el papel que juegan en la conservación del medio ambiente los principales grupos dentro de la sociedad. En este sentido, se señala la necesidad de fomentar el reconocimiento y la participación de las mujeres (Principio 20), los jóvenes (Principio 21), y las comunidades indígenas y tradicionales (Principio 22), para alcanzar el desarrollo sostenible y la protección del medio ambiente.

Señala la necesidad de erradicar la pobreza (Principio 5) y de modificar los patrones de consumo y producción que resultan ambientalmente insostenibles (Principio 8). Reconoce la especial situación en que se encuentran los países en desarrollo (Principio 6) y hace explícito el principio de las responsabilidades comunes pero diferenciadas, frente a la salud y la integridad de ecosistemas, y se fundamenta en las distintas contribuciones a la degradación ambiental global (Principio 7).

A partir de la Declaración de Río ha tenido lugar la iniciación de una etapa que se extiende hasta nuestros días y en la cual la actividad legislativa internacional en materia de protección del medio ambiente no ha perdido intensidad en sus más diversas materias. 


\section{e) Agenda 21}

Su nombre completo es "Programa Global para el Desarrollo sostenible en el Siglo XXI", es el instrumento no vinculante más destacado, significativo e influyente en la esfera del medio ambiente y se ha convertido en el documento orientador en materia de ordenación del medio ambiente en la mayoría de las regiones del mundo. (Programa de las Naciones Unidas sobre el Medio Ambiente, 2000). Sus disposiciones se contienen en 40 capítulos organizados en un preámbulo y cuatro secciones:

1) Dimensión socioeconómica (hábitats, salud, demografía, consumo y producción, etc.);

2) Conservación y administración de recursos naturales (atmósfera, bosques, agua, residuos y productos químicos, etc.);

3) Fortalecimiento del rol de las organizaciones no gubernamentales y de otros grupos sociales como mujeres, jóvenes, grupos empresarios, sindicatos, campesinos, pueblos indígenas y sus comunidades;

4) Implementación de proyectos (financiamiento, instituciones, etc.). Se insiste sobre aspectos del proceso de hacer tratados tales como la futura evolución del derecho internacional sobre desarrollo sostenible y la participación y contribución de todos los países.

\subsection{La Declaración de Johannesburgo}

\subsubsection{Definición}

También es denominada como Cumbre Río+10 pues se realiza una década después de la anterior conferencia, y uno de sus objetivos es evaluar el cumplimiento de los compromisos adquiridos anteriormente y el desafío de lanzar una nueva estrategia que permita avanzar realmente hacia un desarrollo más sostenible, mejorando la calidad de vida y conservación de nuestros recursos naturales.

Según Fajardo (2015) señala que:

La Cumbre Mundial sobre Desarrollo Sostenible, que se celebró en Johannesburgo en 2002, buscaba una mayor implementación de los compromisos de Río. Sus resultados fueron una Declaración final y un Plan de implementación de Johannesburgo que reafirmó el compromiso de la ONU con la plena implementación del Programa 21, junto con el logro de los Objetivos de Desarrollo del Milenio y otros acuerdos internacionales. No aborda delitos ambientales. Sin embargo, en el punto 19 de su Declaración final ${ }^{2}$, se refiere a la delincuencia organizada como una de las amenazas graves para el desarrollo sostenible. (p.12)

\subsubsection{Resultados}

Los resultados de la Cumbre quedaron recogidos en dos importantes documentos adoptados por consenso: la "Declaración de Johannesburgo sobre el Desarrollo Sostenible" suscrita por todos los Jefes de Estado y de Gobierno, y el "Plan de Aplicación de Johannesburgo", éste constituye

\footnotetext{
2 Declaración de Johannesburgo. Punto 19.- "Reafirmamos nuestros votos para poner un particular énfasis en, y dar atención prioritaria a, la lucha contra las condiciones mundiales que presentan severas amenazas al desarrollo sustentable de nuestra gente. Entre estas condiciones están: el hambre crónica; la desnutrición; la ocupación extranjera; los conflictos armados; los problemas de drogas ilícitas; el crimen organizado; la corrupción; los desastres naturales; el tráfico ilícito de armas; el tráfico de personas; el terrorismo; la intolerancia y la incitación al odio racial, étnico, religioso y de otros tipos; la xenofobia; y las enfermedades endémicas, transmisibles y crónicas, en particular el HIV/SIDA, la malaria y la tuberculosis."
} 
el marco global en materia de desarrollo sostenible para los próximos años. El documento cuenta con recomendaciones y objetivos que intentan conciliar el crecimiento económico, la justicia social y la protección del medio ambiente. En la Declaración, los altos dignatarios presentes en la Cumbre se comprometen a edificar una sociedad humana global, equitativa y solícita, consciente de la necesidad de dignidad humana para todos. Además asumen la responsabilidad colectiva de impulsar y fortalecer en los ámbitos local, nacional, regional y global, los fundamentos del desarrollo sostenible: desarrollo económico, desarrollo social y protección ambiental

De igual forma, el Plan pretende poner en práctica los conceptos señalados en la Agenda 21 adoptada en la Cumbre de Río. La Cumbre de Johannesburgo fue una gran oportunidad para que el mundo avanzara hacia un futuro sostenible, en que la gente pudiera satisfacer sus necesidades sin perjudicar el medio ambiente.

Se destaca en el Plan de Aplicación, que para el desarrollo sostenible es fundamental contar con el buen gobierno dentro de cada país y en el ámbito internacional. En el ámbito doméstico las bases para el desarrollo sostenible son: políticas ambientales, sociales y económicas sanas; instituciones democráticas que respondan a las necesidades de los pueblos; el estado de derecho; las medidas contra la corrupción: la igualdad de género y un ambiente apropiado para la inversión. En el ámbito internacional dichas bases son: la paz, la seguridad, la estabilidad, el respeto de los derechos humanos y las libertades fundamentales, incluido el derecho al desarrollo, así como la diversidad cultural.

\subsection{Declaración de Copenhague sobre desarrollo social}

\subsubsection{Definición}

La Declaración de Copenhague reconoce que más mujeres y hombres viven en pobreza extrema y sobre ellos recae una parte desproporcionada de los problemas que conlleva luchar con la pobreza, la desintegración social, el desempleo, la degradación del medio ambiente y los efectos de la guerra; también afirma que la igualdad y la equidad entre el hombre y la mujer es una prioridad para la comunidad internacional y, como tal, debe ser el centro del desarrollo económico y social.

Las razones para celebrarla fueron muchas, pero se resalta el aumento de la prosperidad para algunos, acompañado lamentablemente de un aumento de la pobreza, y el proceso de globalización que es consecuencia del progreso de las comunicaciones, del gran aumento del comercio y de los avances tecnológicos que permite al mismo tiempo, un aumento de la pobreza, desempleo y desintegración; también se han globalizado ciertas amenazas al bienestar del ser humano, como los riesgos ambientales

\subsubsection{Resultados}

En la Cumbre de Copenhague se reconoció que, con el fin de la guerra fría, el concepto de seguridad ha cambiado profundamente. Al decrecer la amenaza de una agresión directa de un Estado contra otro, la seguridad de la gente en su vida diaria - en sus hogares, en sus trabajos, en sus comunidades, en su medio ambiente- se ha convertido en un nuevo imperativo. (Instraw, 1996)

\subsection{Declaración del Milenio de las Naciones Unidas}

\subsubsection{Definición}

Tiene como antecedentes en septiembre de 2000, en el marco de la Cumbre del Milenio, 191 países miembros de la Organización de Naciones Unidas suscribieron la Declaración del Milenio en la ciudad de Nueva York. Este documento contiene ocho compromisos conocidos como los Objetivos 
de Desarrollo del Milenio (ODM-constituyen un marco para que todo el Sistema de Naciones Unidas trabaje en forma conjunta y coherente por un fin común), entre los que destacan erradicar la pobreza y el hambre, mejorar la educación, la equidad de género, la sostenibilidad ambiental, incrementar la salud y fomentar la asociación global para el desarrollo, estos objetivos tienen como fecha límite de consecución el año 2015.

\subsubsection{Resultados}

Lo que nos interesa es principalmente el objetivo 7 que garantiza el sustento del medio ambiente y que comprende: Incorporación de los principios del desarrollo sostenible en las políticas y los programas nacionales y reducir la pérdida de recursos del medio ambiente, haber reducido y haber ralentizado considerablemente la pérdida de diversidad biológica en 2010,y reducir a la mitad, para 2015, la proporción de personas sin acceso sostenible al agua potable y a servicios básicos de saneamiento.

Se enumera seis valores comunes que deben fortalecer dentro de sus políticas, todos los participantes: la libertad, la equidad y solidaridad; la tolerancia, la no violencia; el respeto a la naturaleza, y la responsabilidad compartida.

\subsection{Acuerdo de París}

\subsubsection{Definición}

Streck, Keenlyside y Von Unger (2016) señalan que:

La adopción del Acuerdo de París es un hito en la política internacional sobre el clima y concluye con años de negociaciones casi estancadas. El Acuerdo crea un proceso global de compromiso, seguimiento, ejercicios regulares de inventario y acción cooperativa. Por un lado, representa un paso adelante, superando las muchas divisiones que habían marcado el área de Kyoto: entre desarrollados y en desarrollo. Países, entre las naciones industrializadas dentro del Protocolo y las que están fuera, y entre las que apoyan los mecanismos de mercado y las que se oponen con vehemencia a ellos. Por otro lado, las contribuciones individuales de los países no alcanzan el objetivo climático general, y el riesgo es que el Acuerdo de París siga siendo una cáscara sin suficiente apoyo. El Acuerdo de París es el marco adecuado para abordar el problema de la acción colectiva del cambio climático.(p.1)

Dimitrov (2016) expresa que:

EIAcuerdo de París constituye un éxito político en las negociaciones sobre el clima y la diplomacia tradicional del estado, y ofrece importantes implicaciones para la investigación académica. (...) Es el primer acuerdo global sobre cambio climático que contiene obligaciones de política para todos los países miembros. Es un tratado que consagra dos enfoques ascendentes y descendentes para la gobernanza climática global. Este nuevo acuerdo climático es un acuerdo de laissez-faire entre las naciones que deja el contenido de la política interna a los gobiernos, pero crea obligaciones legales internacionales. Tiene como objetivos principales desarrollar, implementar y fortalecer regularmente las acciones políticas nacionales; y están sujetos a un sólido sistema internacional de transparencia y revisiones globales." (Pp.1-2)

Finalmente, la ambientalista Morris (2016) en cuanto al Acuerdo de París, denota que el objetivo principal "es erradicar las emisiones humanas de gases de efecto invernadero que están causando. Como consecuencia de ello, la atmósfera se calienta, con consecuencias adversas generalizadas, y posiblemente catastróficas. La amenaza del cambio climático se describe como grave, que requiere 
una acción urgente para reducir las emisiones de gases de efecto invernadero, como inversiones a gran escala en adaptación" (p.29).

\subsubsection{Resultados}

Institut de la Francophonie pour le développement durable (2016) en cuanto a los resultados del Acuerdo de París señala que:

brinda a las Partes la oportunidad de utilizar una amplia gama de fuentes, Instrumentos y canales, a través de diversas acciones, teniendo en cuenta el papel crucial de las fuentes públicas. Bajo la presión de los países más vulnerables, (...) el Acuerdo de París pone mayor énfasis en las fuentes públicas en pedir a los países desarrollados que reporten información cada dos años. Teniendo un enfoque de transparencia y coherencia en el apoyo prestado a los países en desarrollo y movilizado por intervenciones públicas (...) (p.23) para reducir en proporción el gas invernadero, a consecuencia de ello, erradicar el cambio climático en la Comunidad Internacional.

\section{CONCLUSIONES}

1) La preservación ambiental es un asunto de materia global porque los problemas que acontecen en el medio ambiente son principalmente de carácter internacional, debido a una crisis de sostenibilidad ambiental que acontece alrededor del mundo, no siendo ajeno por parte de todos los seres vivientes en la tierra, por ello la Comunidad Internacional se ha visto en la necesidad de implementar instrumentos jurídicos internacionales que protejan el medio ambiente desde todas sus concepciones.

2) La Declaración de Estocolmo es un instrumento de carácter programático, sus principios carecen de fuerza vinculante para los Estados, pero, desarrollaron una influencia en la práctica internacional medio ambiental posterior. Por tanto, marca el inicio de una nueva conciencia de la sociedad internacional en orden a la protección del medio ambiente.

3) Desde la Cumbre de la Tierra se han logrado algunos cambios significativos como: una mayor concienciación internacional y local pasando por la implementación y perfeccionamiento de infraestructuras gubernamentales apropiadas, por la cooperación del sector privado, por la propagación de organizaciones no gubernamentales de apoyo medioambiental y por una relativa, pero importante sensibilización de la opinión pública; sin embargo, parece haberse congelado la promoción y ejecución del desarrollo sostenible y sus tres vertientes interdependientes, principal enfoque de la última Cumbre mundial en Johannesburgo.

4) Una de las declaraciones más importantes del derecho ambiental es el Acuerdo de París, que plantea medidas para reducir los efectos de gases invernaderos para poder adaptarse a los efectos del cambio climático e incrementar desarrollo que generen bajas de estas mismas, para ello cada Estado miembro que ha ratificado dicho acuerdo internacional, ha ido desarrollando en cada uno de sus Estados la aplicación de políticas públicas que reduzcan los gases de efecto invernadero; y mejorar la capacidad e infraestructura de su territorio para adaptarse a los efectos del calentamiento global.

\section{REFERENCIAS}

- $\quad$ Andaluz, C. (2006). Manual de Derecho Ambiental. Lima: Proterra.

- Atilio, J. (2007). Tratado de Derecho Ambiental. Buenos Aires: Ediciones Jurídicas.

- Bustamante, J. (1995). Derecho Ambiental. Buenos Aires: Abeledo Perrot. 
- Dimitrov, R. (2016). The Paris Agreement on Climate Change: Behind Closed Doors. En: Global Environmental Politics 16:3, Pp. 1-11.

- Fajardo, T. (2015). International Environmental Law and Environmental Crime: An Introduction. Granada: Universidad de Granada.

- Foy, P. y Novak, F. (2003). Derecho Internacional Ambiental. (1ª. Ed.) Lima: Pontificia Universidad Católica del Perú.

- Institut de la Francophonie pour le développement durable. (2016). Accord de Paris sur le climat. Obtenido en : https://www.climatechance2016.com/uploads/media/57e533970be47. pdf

- Jaquenod, S. (1991). El Derecho Ambiental y sus principios rectores. (3era.Ed). Madrid: Dykinson.

- Lozano, B. (2007). Derecho Ambiental Administrativo. (10ª ed). Madrid: Dykinson.

- Morris, J. (2016). The Paris Agreement: An Assessment. En: Reason Foundation Policy Brief No. 133, Pp. 1-31.

- Noticias de Instraw, Mujer y Desarrollo, Edición Especial 1996, №24/25. Obtenido en: https://www.redmujer.org.ar/

- Russell, K. (2014). Mechanisms for Global Agreements. Obtenido en: https://www. bdlaw.com/content/uploads/2018/06/mechanisms-for-global-agreements-r-lamotte-abainternational-law-guide.pdf

- $\quad$ Streck, C, Keenlyside, P. y Von Unger, M. The Paris Agreement: A New Beginning. En: Journal for european environmental \& planning law 13, Pp. 3-29.

- Strong, M. (2001). Hacia Río de Janeiro 1992. En: Foro del Desarrollo, Vol.19, №2.

- Universidad de Melbourne. (2018) Public International Law: International Environmental Law Obtenido en: http://unimelb.libguides.com/internationallaw/environmental

Fecha de recepción: 30 de abril de 2019

Fecha de aceptación: 16 de junio de 2019 